\title{
Unit per Millimole
}

National Cancer Institute

\section{Source}

National Cancer Institute. Unit per Millimole. NCI Thesaurus. Code C92618.

An arbitrary unit of substance concentration equal to the concentration at which one

millimole of a mixture contains one unit of a substance. 